\title{
nAnOINK
}

NanoFabrication Systems

\section{Direct deposition of micro- and nanoscale hydrogels using Dip Pen Nanolithography (DPN)}

Dip Pen Nanolithography ${ }^{\circledast}\left(\mathrm{DPN}^{\mathrm{TM}}\right)$ is the controlled delivery of material from nanoscale tips to a surface. Its advantages over other lithography techniques include low operating costs, high resolution, nanoscale registry and multiplexed deposition capabilities. With Nanolnk's latest nanolithography tools and new biocompatible patterning protocols, any scientist can easily and rapidly create arbitrary hydrogel patterns with subcellular feature sizes to be used for cell adhesion, cell migration and drug delivery studies.

DPN is a technique in which multiple materials are patterned onto a surface using nanoscale tips ${ }^{1}$. Nanolnk's nanolithography tools offer several important advantages over other instruments that rely on more conventional lithography techniques. DPN instruments can reproduce virtually any feature that can be created using high-cost clean-room equipment, all at ambient temperature and pressure. Such conditions are essential for creating substrates that are permissive to cell culture. With tip-based nanolithography, biocompatibility need not be sacrificed to achieve nanoscale precision and registry ${ }^{2}$.
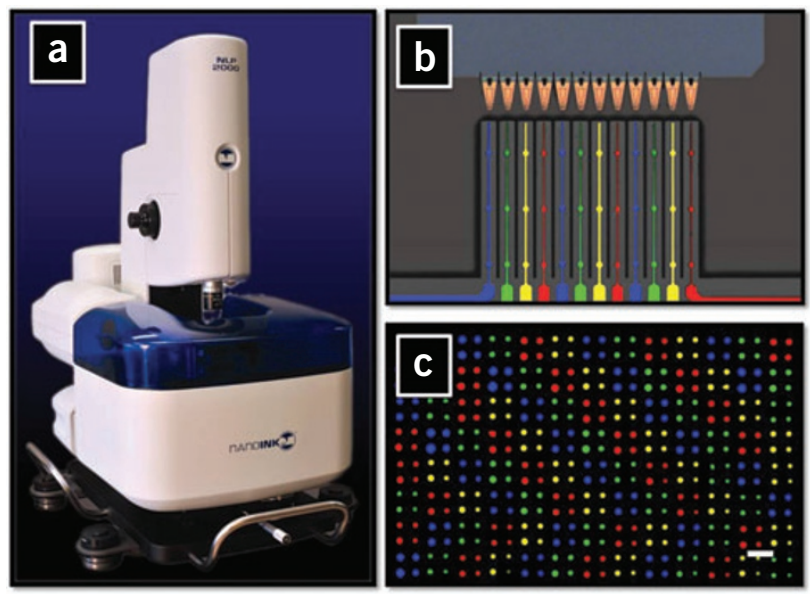

Figure 1 (a) NLP 2000 System. (b) Optical image of Nanolnk's M-type cantilever tip array overlaid with a fluorescence image of a 12-microchannel 'Inkwell'. The Inkwell contains four separate dye-labeled lgG antibodies. (c) Fluorescence image (20x objective) of the same four antibodies deposited simultaneously using the NLP 2000 System (scale bar, $10 \mu \mathrm{m}$ ).

\section{Paul L Stiles}

Nanolnk, Inc., Skokie, Illinois, USA.

Correspondence should be addressed to P.L.S. (pstiles@nanoink.net).
The NLP 2000 System is Nanolnk's latest DPN instrument (Fig. 1a). It is designed specifically for researchers interested in creating complex, multicomponent substrates for use in biological experiments. The system is designed to control the leveling and translational positioning of multiple nanoscale tips. The tips are loaded with printing materials using Nanolnk's microfluidic chips, or 'Inkwells', as shown in Figure 1b. The figure is a combination of two images, one of an Inkwell filled with four different dye-labeled protein solutions and the other a topdown view of Nanolnk M-type cantilever tip arrays. They are shown together to demonstrate how each tip lines up with a microchannel, enabling simultaneous loading of separate materials on each tip. After dipping into the Inkwells, the tips can be moved over to a surface, where they will transfer controlled amounts of the desired material, creating features as small as $50 \mathrm{~nm}$ and as large as $5 \mu \mathrm{m}$. The technique is analogous to multiple pens being used in a printing process-hence the name 'dip pen nanolithography'. Figure 1c is a fluorescence image showing the multiplexed deposition of four different proteins. The figure demonstrates that even when submicron feature size is not required (the scale bar is $10 \mu \mathrm{m}$ ), the nanoscale precision of the NLP 2000 System's translation stages is essential for the creation of wellordered patterns.

\section{Hydrogel depositions}

To further address the needs of biomaterials researchers, Nanolnk has developed a new biocompatible PEG-based polymer that can be crosslinked after deposition to form nano- or microscale hydrogel patterns. These gels can act as repulsive barriers for studying controlled cell migration, or they can be combined with separate functional polymers for research into cell-adhesion properties ${ }^{3}$.

Hydrogels are three-dimensional cross-linked polymer networks that readily absorb water, giving them physical characteristics very similar to those of natural tissue. They have become extremely useful 
in biomaterials research. In their pure form, hydrogels resist cell and protein binding while maintaining many of the mechanical properties of soft tissue. PEG hydrogels' protein-binding and mechanical properties can easily be altered with only a few simple chemical modifications. Combining this chemical versatility with the ability to quickly and easily deposit micro- and nanoscale patterns opens up many new research possibilities.

The first step in the deposition of subcellular hydrogel features is to mix a hydrogel precursor (liquid-phase PEG-dimethacrylate, or PEG-DMA) with a small amount of a photoinitiator. DPN tips are loaded with the hydrogel precursor and then used to deposit a pattern onto the substrate. After deposition, the substrate is removed from the DPN instrument and cross-linked in a UV chamber. Nanolnk has demonstrated that this method can create hydrogel arrays with individual features smaller than $50 \mathrm{~nm}$ and as large as $10 \mu \mathrm{m}$. Feature size is easily controlled by adjusting PEG-DMA molecular weight, deposition temperature and the amount of time that the DPN tip is in contact with the substrate. Figure 2 shows representative images of patterns created by directly deposited hydrogels. Figure $\mathbf{2 a}$ is an atomicforce microscope (AFM) image of a high-density hydrogel array, Figure $\mathbf{2 b}$ is a fluorescence image of four different dye-labeled hydrogels patterned simultaneously using multiple tips, and Figure 2c shows an array of FITC-labeled avidin encapsulated in a hydrogel matrix.
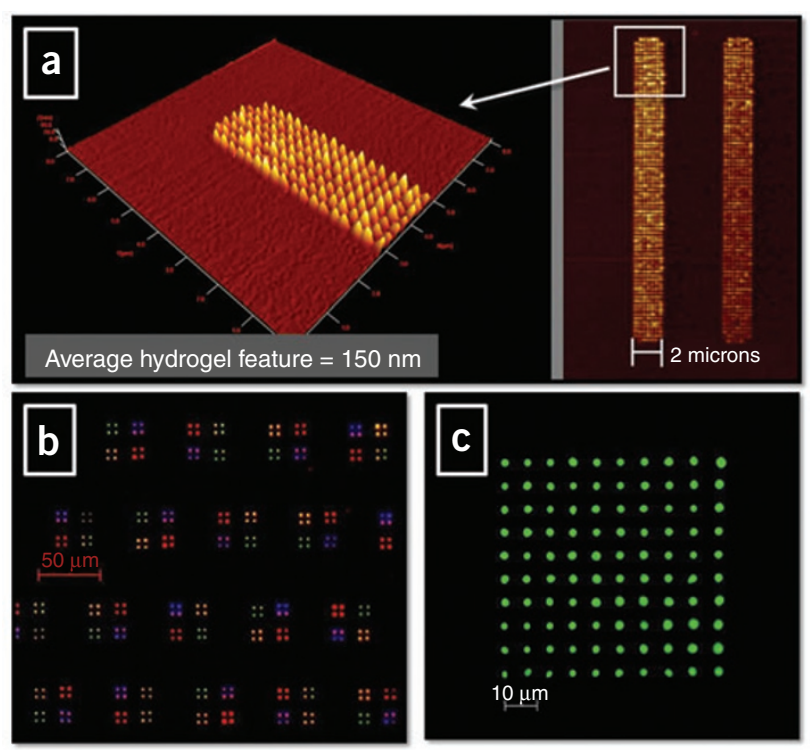

Figure 2 (a) AFM image of high-density hydrogel array. (b) Fluorescence image of four different dye-labeled hydrogels. (c) Fluorescence image of FITC-labeled avidin encapsulated in a hydrogel matrix.

\section{Functionalized hydrogels}

Tip-based printing is never limited to a single type of deposition material, and this is especially true for hydrogel printing. For instance, the PEG-DMA polymer discussed above can be mixed with a different PEG-based polymer, namely a four-arm PEG thiol (Fig. 3). After loading of DPN tips and deposition of the PEG-DMA/four-arm PEG thiol material, the substrate is placed under a UV light source for cross-linking. The reaction proceeds as a mixed-mode step and chain-growth polymerization process: the PEG-DMA reacts with other PEG-DMA molecules via a chain polymerization, and the PEG-DMA reacts with the thiol functional groups on the four-arm PEG thiol via a step-growth mechanism. Because the chain reaction is much faster than the step-growth reaction, all of the PEG-DMA will be consumed before the entire four-arm PEG thiol has time to completely react, resulting in a hydrogel with exposed thiol groups that can be used in subsequent reactions ${ }^{4}$. The reaction scheme and resultant data are shown in Figure 3.

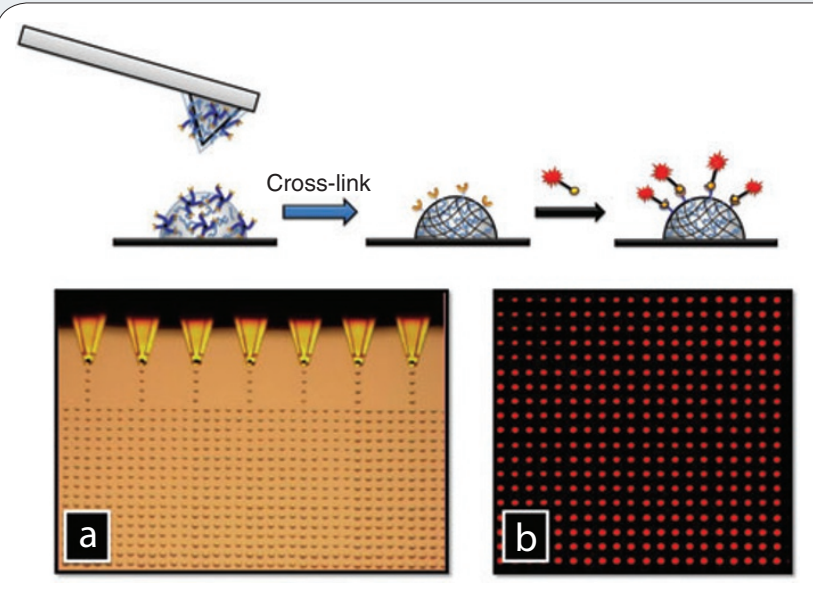

Figure 3 Deposition, cross-linking and tagging scheme. (a) Screen capture of mixed polymer deposition in progress. (b) Fluorescence image of functionalized hydrogel after reaction with rhodamine-conjugated maleimide.

There are several advantages of creating functionalized hydrogels. First, PEG-based hydrogels are resistant to protein binding, ensuring that any protein bound to the surface of the hydrogel in a secondary reaction will attach only to the exposed thiol groups. Second, the number of exposed thiol groups on the hydrogel can be controlled by altering the ratio of the PEG-DMA to the four-arm PEG thiol in the initial hydrogel precursor. Lastly, the different ratios of polymers in the different hydrogel precursors will all be deposited at the same rate because the bulk of the polymers are exactly the same, namely PEG. The combination of all of these advantages gives the NLP 2000 System the ability to create arbitrary surface gradients of virtually any protein. Nanolnk's multiplexed deposition methods can deposit multiple polymer ratios simultaneously, in a single experiment, and then tag any protein to the different functionalized hydrogels using standard proteomic cross-linking techniques.

1. Salaita, K., Wang, Y.H. \& Mirkin, C.A. Applications of dip-pen nanolithography. Nat. Nanotechnol. 2, 145-155 (2007).

2. Yousaf, M.N. Model substrates for studies of cell mobility. Curr. Opin. Chem. Biol. 13, 697-704 (2009).

3. Miller, J. \& West, J. Biomimetic hydrogels to support and guide tissue formation. in Micro and Nanoengineering of the Cell Microenvironment: Technologies and Application (eds. Khademhosseini, A. et al.) Ch. 6, 101-120 (Artech House, Boston, 2008)

4. Rydholm, A.E., Held, N.L., Benoit, D.S., Bowman, C.N. \& Anseth, K.S. Modifying network chemistry in thiol-acrylate photopolymers through postpolymerization functionalization to control cell-material interactions. J. Biomed. Mater. Res. 86, 23-30 (2008).

This article was submitted to Nature Methods by a commercial organization and has not been peer reviewed. Nature Methods takes no responsibility for the accuracy or otherwise of the information provided. 